\title{
Characterizing Multiphoton Excitation Using Time-Resolved X-ray Scattering
}

\author{
Philip H. Bucksbaum $\odot,{ }^{1,2,3}$ Matthew R. Ware, ${ }^{3}$ Adi Natan, ${ }^{3}$ James P. Cryan, ${ }^{3,4}$ and James M. Glownia ${ }^{3,4}$ \\ ${ }^{1}$ Department of Physics, Stanford University, Stanford, California 94305, USA \\ ${ }^{2}$ Department of Applied Physics, Stanford University, Stanford, California 94305, USA \\ ${ }^{3}$ Stanford PULSE Institute, SLAC National Accelerator Laboratory, Menlo Park, California 94025, USA \\ ${ }^{4}$ Linac Coherent Light Source, SLAC National Accelerator Laboratory, \\ Menlo Park, California 94025, USA
}

(Received 7 November 2019; revised manuscript received 15 January 2020; accepted 7 February 2020; published 17 March 2020)

\begin{abstract}
Molecular iodine is photoexcited by a strong 800-nm laser driving several channels of multiphoton excitation. The motion following photoexcitation is probed using time-resolved $\mathrm{x}$-ray scattering, which produces a scattering map $S(Q, \tau)$. Temporal Fourier-transform methods are employed to obtain a frequency-resolved x-ray-scattering signal $\tilde{S}(Q, \omega)$. Taken together, $S(Q, \tau)$ and $\tilde{S}(Q, \omega)$ separate different modes of motion so that mode-specific nuclear oscillatory positions, oscillation amplitudes, directions of motion, and times may be measured accurately. Molecular dissociations likewise have a distinct signature, which may be used to identify both velocities and dissociation time shifts and also can reveal laser-induced couplings among the molecular potentials.
\end{abstract}

DOI: 10.1103/PhysRevX.10.011065

\section{INTRODUCTION}

Intense ultrafast-laser irradiation of molecules leads to nonlinear processes that can include multiphoton absorption, impulsive stimulated Raman scattering [1,2], and hyper-Raman fluorescence [3,4]. At still higher intensities, molecules undergo bond softening [5], tunnel ionization, charge-resonant-enhanced ionization [6], and multiple ionization [7]. All of these phenomena have been studied extensively using the tools of fluorescence, coherent light scattering, and photoelectron spectroscopies; however, these optical-based methods cannot measure directly the subangstrom displacements and femtosecond motion of the molecular nuclei in response to the light. Here we employ femtosecond hard x-ray scattering, a new method that fills this gap in our measurement capabilities $[8,9]$.

We choose one of the simplest well-studied systems, molecular iodine irradiated with ultrafast pulses of focused 800-nm radiation [10] (see also Fig. 1). This wavelength is close to resonance with transitions to the weakly absorbing $A$ state in the molecule. We work in the impulsive vibrational regime with 70 -fs pulses focused to about $10^{13} \mathrm{~W} / \mathrm{cm}^{2}$. The absorption fraction is $6 \%$, as measured by depletion of the ground-state diffraction scattering pattern. At this intensity, both linear and nonlinear photon

Published by the American Physical Society under the terms of the Creative Commons Attribution 4.0 International license. Further distribution of this work must maintain attribution to the author(s) and the published article's title, journal citation, and DOI.
Subject Areas: Atomic and Molecular Physics, Chemical Physics

processes are present in the system. We observe a number of nonlinear processes that are described below. We do not observe significant ionization, a six-photon process.

For example, one prominent nonlinear process excited under these conditions is impulsive stimulated Raman scattering (ISRS) [2], a coherent two-photon redistribution of the initial population of the molecule into an oscillating vibrational wave packet in the ground electronic state [Fig. 1(a)]. This ground-state motion can be tracked through changes to the $\mathrm{x}$-ray scattering pattern [11]. In iodine, this Raman cross section is extremely strong. Furthermore, the ground-state potential energy surface is nearly parabolic in iodine, so the quantum wave packet generated by ISRS is not highly dispersive and persists for dozens of oscillations.

The experimental setup, data collection, and data preparation used in analysis are all described in Sec. II. The separate

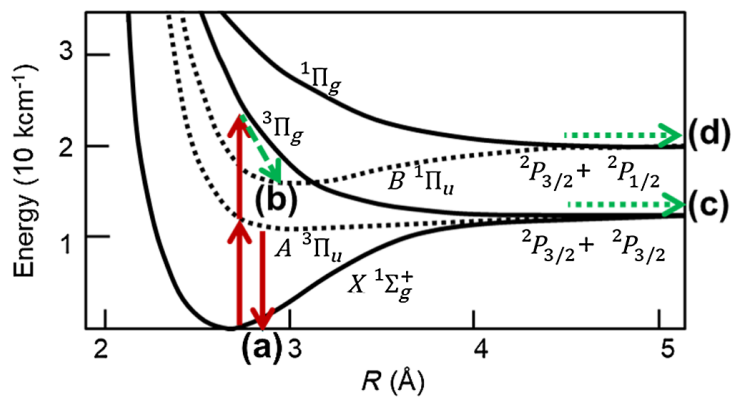

FIG. 1. The relevant potential energy levels in $\mathrm{I}_{2}$, together with the nonlinear processes observed in this experiment. (a) Impulsive simulated Raman scattering. (b) Spontaneous hyper-Raman scattering. (c),(d) Dissociation following two-photon absorption. 
analysis of each nonlinear photoinduced process detected and tracked by the time-resolved $\mathrm{X}$ rays is described in Sec. III, with subsections devoted to each topic. A table at the conclusion of the paper summarizes each nonlinear phenomenon, its measurement method, and when possible, comparisons to theory.

\section{IODINE EXPERIMENT}

\section{A. Time-resolved x-ray scattering}

To view the oscillations and other nonlinear laserinduced motion, we employ time-resolved $x$-ray scattering (TRXS) using femtosecond $\mathrm{X}$ rays from the LCLS $\mathrm{x}$-ray free-electron laser [11,12]. X rays of $1.4 \AA, 40$ fs scatter from the photoexcited molecules at an adjustable time delay, and the angle of scattering is recorded on a twodimensional detector. (See Ref. [13] for details on the timescan data-collection methodology.) A typical scattering pattern is shown in Fig. 2.

Each section of this scattering pattern has its own time dependence. For example, the integrated signal for one of the tiles shown in Fig. 2 oscillates with time delay as shown in Fig. 3. The Fourier transform of these data shows several distinct peaks in Fig. 4. Based on our knowledge of the potential energy surfaces in iodine, we can confidently assign the larger peak at $40 \times 10^{12} \mathrm{rad} / \mathrm{s}$ to the ISRS process in Fig. 1(a) and more tentatively assign the smaller peak at $25 \times 10^{12} \mathrm{rad} / \mathrm{s}$ to process in Fig. 1(b). Figures 3 and 4 are the kind and quality of information that has long been available using standard one-dimensional pump-probe methods such as time-resolved fluorescence. TRXS provides this information for each scattering angle $Q$, and we can use this to obtain information about structure and motions.

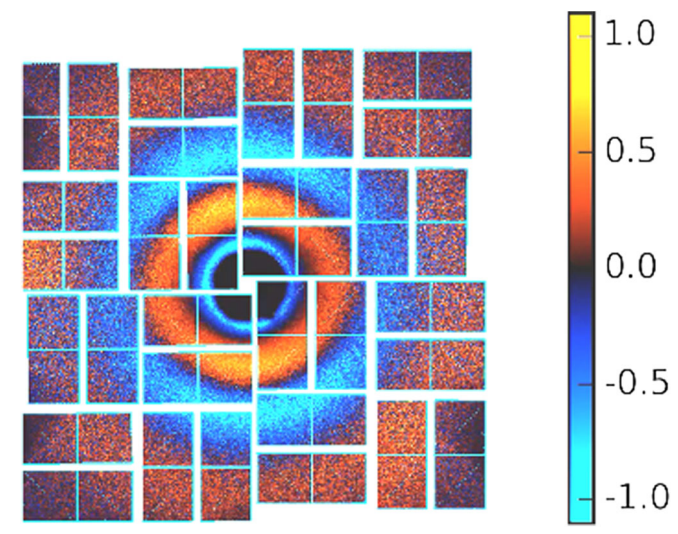

FIG. 2. Change in the X-ray scattering distribution recorded for the X-ray pulse delayed by $40 \mathrm{fs}$ following intense laser excitation [14]. Following established procedures, this image is corrected to remove the polarization dependence due to Thompson scattering and subtract the background of molecules that are not excited. Therefore, the features seen here are due to the changes in scattering brought about by intense laser excitation.

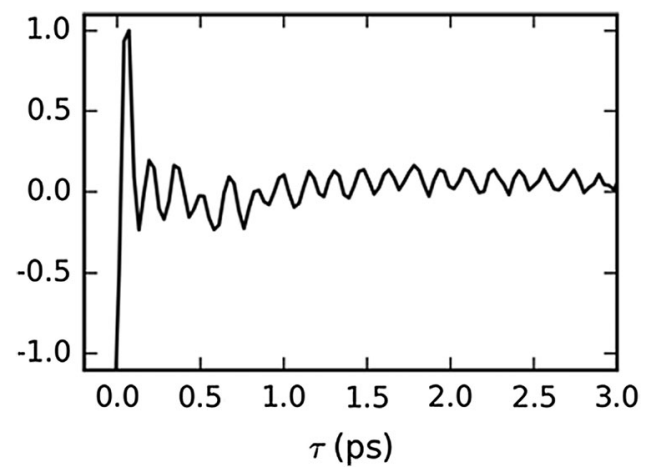

FIG. 3. Signal vs time delay for a single tile in Fig. 2. The tile chosen is the second row, fourth column.

The obvious inversion symmetry in Fig. 2, which is guaranteed by the inversion symmetry of the target gas ensemble, can be used to rewrite the three-dimensional scattering function $S(\vec{Q}, \tau)=S(Q, \cos \theta, \tau)$ as a Legendre series with only even terms $S_{l=0,2, \ldots,}(Q, \tau)$, where

$$
S_{l}(Q, \tau)=\frac{2 l+1}{2} \int_{-1}^{1} P_{l}(\cos \theta) S(Q, \cos \theta, \tau) d(\cos \theta) .
$$

The first two terms in this series, the isotropic term and the term that varies as $P_{2}(\cos \theta)=\left(3 \cos ^{2} \theta-1\right) / 2$, contain most of the data. Figure 5 shows the $l=0$ isotropic (i.e., angle-integrated) term. The oscillating pattern that dominates Fig. 3 is still present, but now it can be viewed for every value of $Q$.

\section{B. Frequency-resolved x-ray scattering}

The same extension to all $Q$ 's can be performed for the power spectrum in Fig. 4. Here it is useful to retain the full complex Fourier transform $\tilde{S}(Q, \omega)$ shown in Fig. 6 . We show that the relative phase for different values of $Q$ has physical meaning [14].

A real advantage to displaying the data in this way is that each process shown in Fig. 1 is cleanly separated from the others [15]. This is even more easily seen in the power

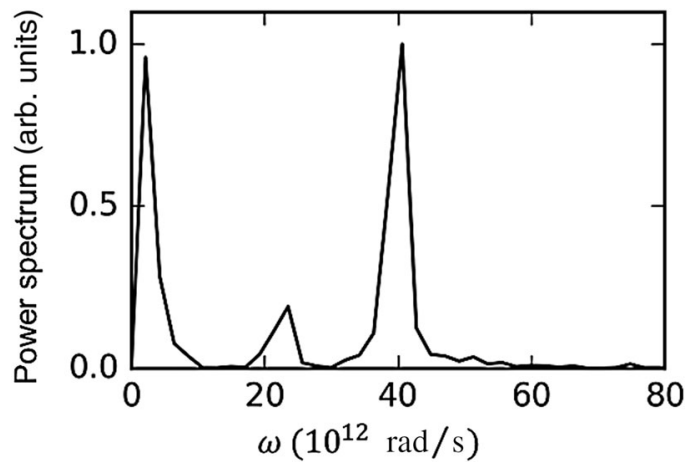

FIG. 4. Power spectrum (squared amplitude of the Fourier transform) of Fig. 3. 


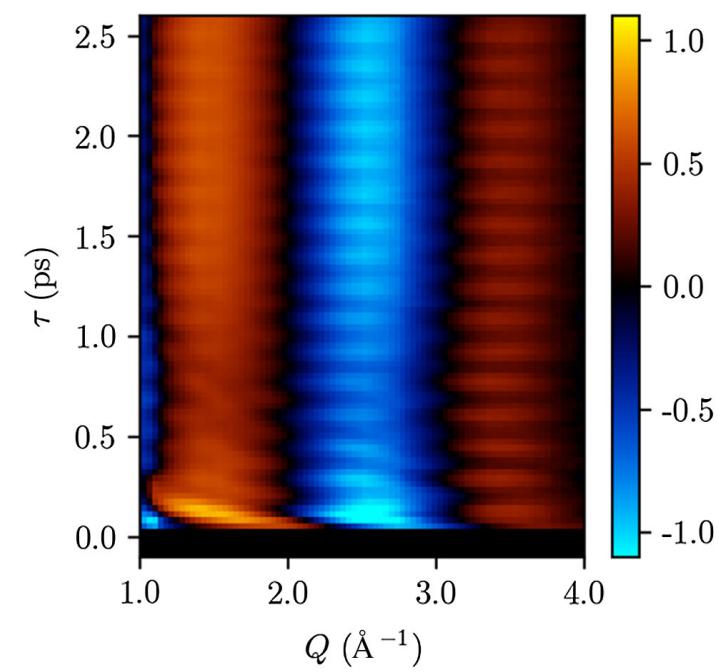

FIG. 5. $S_{0}(Q, \tau)$ : Scattering data projected on to $P_{0}(\cos \theta)$ minus unpumped scattering.
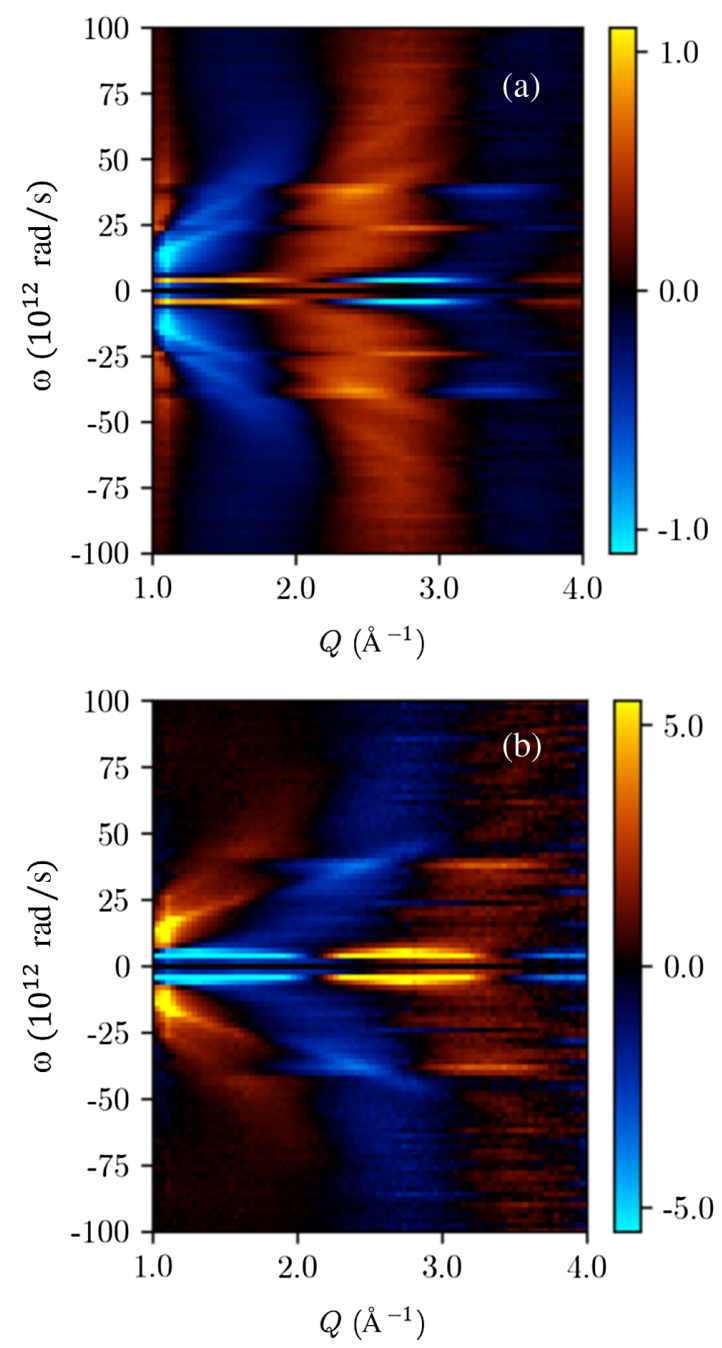

FIG. 6. Fourier transform of $S_{0}(Q, \tau)$ in Fig. 5 to form (a) $\operatorname{Re}\left\{\tilde{S}_{0}(Q, \omega)\right\}$ and (b) $\operatorname{Im}\left\{\tilde{S}_{0}(Q, \omega)\right\}$.

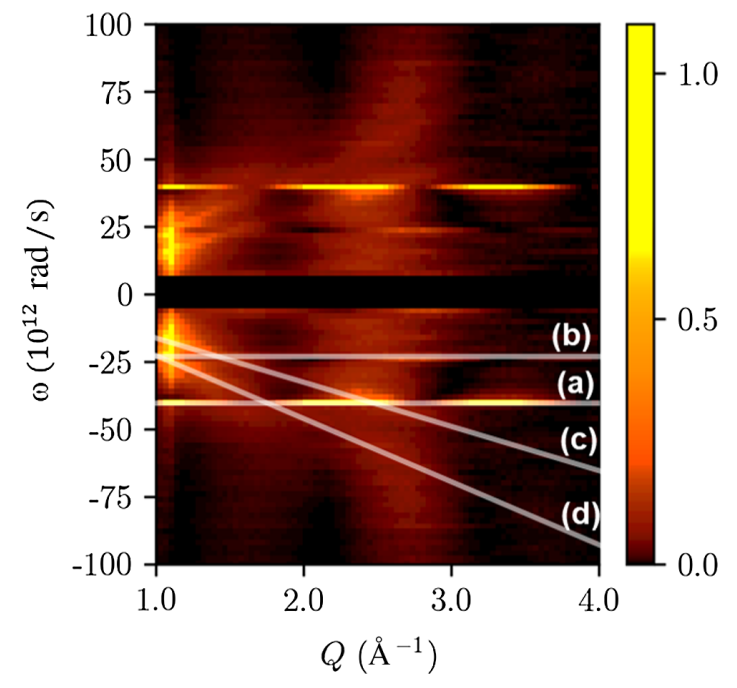

FIG. 7. The power spectrum $\mathcal{P}_{0}(Q, \omega)=\left|\tilde{S}_{0}(Q, \omega)\right|^{2}$. The lowfrequency part of the plot $\left(\omega<5 \times 10^{12} \mathrm{rad} / \mathrm{s}\right)$ is darkened to help enhance the contrast. Nonlinear processes shown in Fig. 1 appear as straight lines when the data are displayed in this way. These excitations are identified in the lower half-plane of the figure. (a) Impulsive simulated Raman scattering. (b) Spontaneous hyper-Raman scattering. (c),(d) Dissociation.

spectrum $\mathcal{P}_{0}(Q, \omega)=\left|\tilde{S}_{0}(Q, \omega)\right|^{2}$ shown in Fig. 7. We now consider each of these features in the data to measure the structural information that is associated with each kind of motion.

\section{ANALYSIS}

\section{A. Impulsive stimulated Raman scattering}

The strongest high-frequency feature in Fig. 7 is the bright narrow horizontal line at $\omega \approx \pm 40 \times 10^{12} \mathrm{rad} / \mathrm{s}$. (The data at angular frequencies below $5 \times 10^{12} \mathrm{rad} / \mathrm{s}$ correspond to rotational wave packets that evolve over picoseconds and do not concern us here.) This line is associated with ISRS redistribution among the nearlyevenly-spaced vibrational levels of the ground state of the iodine molecule. A measurement of this feature of Fig. 7 integrated over $Q$ yields a value of $|\omega|=40.3 \pm$ $1.0 \times 10^{12} \mathrm{rad} / \mathrm{s}$ compared with a literature value of $40.42 \times 10^{12} \mathrm{rad} / \mathrm{s}(6.43 \mathrm{THz})$ [16]. Again, this information can be obtained by all-optical nonlinear spectroscopic methods.

The added advantage of ultrafast $\mathrm{x}$-ray probes of this system is to yield correlated spatial and motion information to accompany the transient spectra. Data from x-ray scattering can answer several questions about the dynamics of motion in the ISRS process in iodine: Where is the motion within the molecule that is responsible for the transient spectra? What is the amplitude of this motion? What phase, i.e., when initially excited, does the molecule first contract or first expand? Here we show how these 


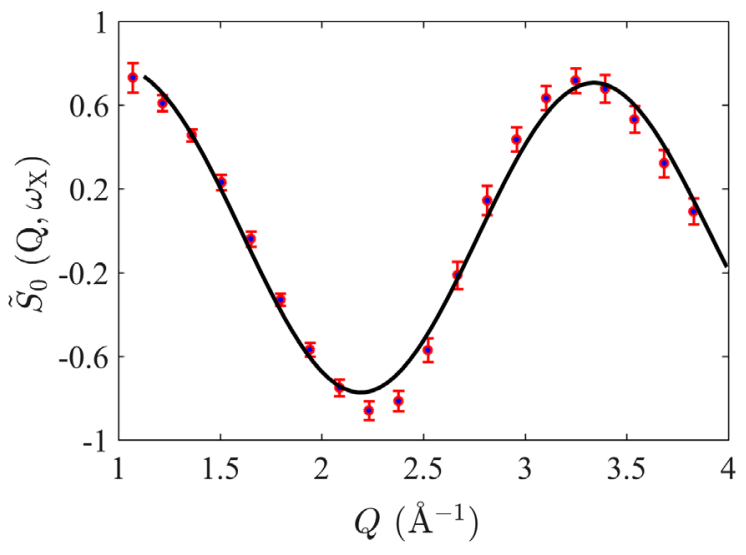

FIG. 8. Points: Data from $\operatorname{Im}\left\{\tilde{S}_{0}(Q, \omega)\right\}$ in Fig. 6 in $0.15 \AA^{-1}$ bins for $\omega=40.3 \pm 1.0 \times 10^{12} \mathrm{rad} / \mathrm{s}$. Line: Fit using Eq. (2) finds the vibrational mode centered at $R=2.79 \pm 0.07 \AA$ with vibrational motion amplitude $A=0.164 \pm 0.01 \AA$. For comparison, the iodine ground state has an equilibrium separation of $2.67 \AA$, and the molecular potential well width varies from $0.173 \AA$ at $v=1$ to $0.335 \AA$ at $v=5$ [17].

questions may be answered from the data as displayed in Figs. 5-7.

The $Q$ dependence of the ISRS feature in Figs. 6 and 7 reveals the internuclear separation of the iodine atoms where the motion occurs. This connection can be seen in the lineout displayed in Fig. 8 taken from $\operatorname{Im}\left\{\tilde{S}_{0}(Q, \omega)\right\}$. The scattering signal expected from an ensemble of diatomic molecules with constant internuclear distance $R_{0}$ is [14]

$\tilde{S}_{0}\left(Q, \omega_{0}\right)=\frac{A}{2 R_{0}}\left(\cos Q R_{0}-\sin c Q R_{0}\right) \delta\left(\omega-\omega_{0}\right)$.

This function predicts zeros in the scattered signal at $Q R_{0}=0,4.5,7.7,10.9$, and so on, and these can be used to determine $R_{0}$ if the amplitude of oscillation $A$ is small.

For finite $A$ but still smaller than $R_{0}$, the scattering pattern of $\tilde{S}\left(Q, \omega_{0}\right)$ in Figs. 6 and 7 is a similar modulation due to $R_{0}$ but contained within a slowly varying envelope determined by $A[18]$ :

$$
\tilde{S}_{0}\left(Q, \omega_{0}\right) \propto \frac{1}{Q} \int_{0}^{Q} d Q^{\prime} \sin \left(Q^{\prime} R_{0}\right) J_{1}\left(Q^{\prime} A\right)
$$

A fit to Eq. (3) in Fig. 8 yields a value of $R_{0}=2.79 \pm$ $0.07 \AA$, the average interatomic spacing of the ISRS motion in the laser-excited system. The expected value for $R_{0}$ depends on the vibrational redistribution brought about by the impulsive Raman interaction, which strongly favors $\delta v= \pm 1$. The average iodine atomic spacing for the lowlying vibrational levels is $2.68 \AA$, close to our measured value.
Two features of this measurement are noteworthy: First, we arrive at this value through a de novo analysis based on Eq. (3) without using any prior knowledge or theory of the structure of iodine. Second, this analysis is isolated on the ISRS motion, and allows us to filter out any background processes and even to filter out the portion of our x-ray scattering from iodine molecules that are not excited.

The effect of the finite amplitude of motion is more difficult to discern in this plot. The amplitude creates a spread of $Q$ values and leads to decay of the ISRS feature with increasing $Q$. Our collection geometry restricts the scattering vector $Q$ to less than $4 \AA^{-1}$, so small $A$ leads to a small but noticeable decay of the oscillation amplitude with $Q$, leading to a best-fit value of $A=0.164 \pm 0.01 \AA$. This fit value is reasonable for a vibrational wave packet in iodine making excursions between the classical turning points in the ground-state potential well for $v \approx 4$ [17]. This fit, however, assumes that the oscillation amplitude is constant over the period of observation of several picoseconds, and we know that this is not the case.

A more obvious view of this amplitude of oscillation is in the time-domain data in Figs. 5 and 9. As the atomic separation changes, so does the corresponding scattering pattern. For small amplitude motion, the amplitude of the observed $Q$-space oscillation is approximately linearly proportional to the amplitude of the oscillation in real space, and $\pi$ radians out of phase, since stretching the bond decreases the angle of maximum scattering. The amplitude of this feature is also proportional to the relative population of oscillating molecules, but if the motion of interest is the dominant mode in the data shown in Fig. 5 (i.e., the excitation fraction to this mode is nearly unity), then the oscillation amplitude can be seen easily in that figure.

Figure 9 shows that the scattering pattern $S(Q, \tau)$ in the vicinity of the zero crossing at $Q=2 \AA^{-1}$ oscillates in $Q$ with a peak-to-peak amplitude that varies from $5 \%$ of the central $Q$ value near $\tau=0$ to about $2 \%$ over $1-3$ ps. This behavior implies oscillation amplitudes that start at $0.14 \AA$

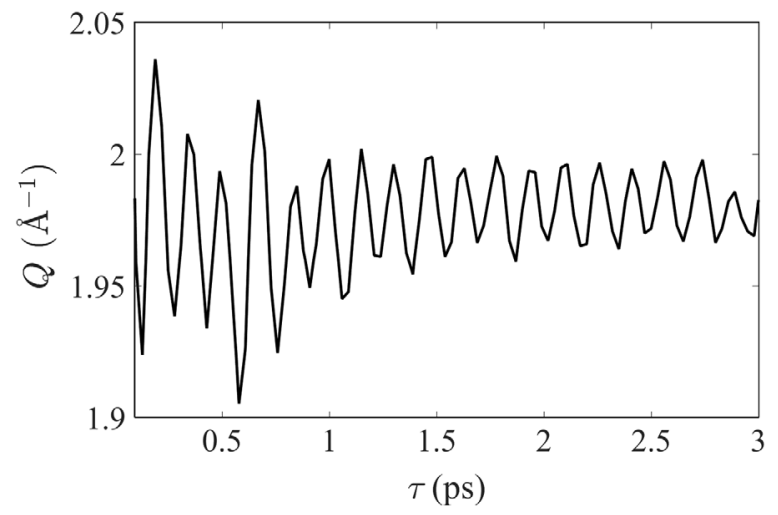

FIG. 9. Oscillation of the node in $S(Q, \tau)$ (Fig. 5) in the vicinity of $2 \AA^{-1}$. 
and drop to $0.05 \AA$ Aver the duration of the observation. The discrepancy from Fig. 8 reflects how TRXS and the frequency-resolved x-ray scattering (FRXS) highlight different features of the scattering. In FRXS (Fig. 8), the changes in bond length modulate the envelope of scattering oscillations in $Q$, while in TRXS (Fig. 9), we analyze how bond length affects the diffraction at a single $Q$. The observed decay in the wave-packet signal in Fig. 9 has also been noted in experiments using optical probes and attributed to rotational dispersion of the ensemble molecular alignment [19]. We conclude that the value found in the fit to $\tilde{S}(Q, \omega)$ is a frequency-filtered periodic component of a more complicated motion.

The phase of the ISRS oscillation may also be read directly from Fig. 5 by noting that at $\tau=0$, the initial oscillation is moving toward smaller $Q$. This shows that immediately following the ISRS excitation by the laser, the molecule starts to expand. Another way to recover this information is by comparing the real and imaginary parts of the temporal Fourier transform $\tilde{S}(Q, \omega)$ in Fig. 6. This quantity is the Fourier transform of $S(Q, \tau)$, so the phase $\delta$ of an oscillation feature

$$
Q(\tau)=Q_{0}+Q_{1} \cos \left(\omega_{0} \tau+\delta\right)
$$

is a measure of the starting conditions of motion for that frequency and scattering vector.

For example, a feature oscillating like Eq. (4) with $\delta=0$ begins its motion at a turning point. This motion would appear as a purely real feature centered at $Q_{0}$ in $\tilde{S}(Q, \omega)$. The real and imaginary parts of $\tilde{S}(Q, \omega)$ displayed in Fig. 6 show that the $40 \times 10^{12} \mathrm{rad} / \mathrm{s}$ feature in $\tilde{S}(Q, \omega)$ is almost purely imaginary. We measure $\delta=290^{\circ}$ at $Q=2.4 \AA^{-1}$. This means that, as we have already seen, $Q$ is near the center of its oscillation and contracting at $\tau=0$, and therefore, the molecule expands at $\tau=0$.

For completeness, we note that the oscillation amplitude and phase can also be measured directly by transforming Fig. 5 to a scattering plot $S(r, \tau)$ of the spatial autocorrelation vs time using spatial Fourier-transform techniques. Unfortunately, the signal in the appropriate range of $r$ is congested and suffers from poor resolution in these experiments, so this method is difficult in practice.

Information about the motion of a molecule just after excitation is inferred from the signal in all-optical pumpdump protocols, where population is driven between different potential energy surfaces. But in such cases, the conclusion relies on knowledge of the potential surfaces in advance. X-ray scattering does not rely on this prior knowledge and can show details of motion purely on the ground-state surface. This may provide more useful connections to collision chemistry.

So far, we consider only the strongest nonlinear excitation in the iodine ISRS. Figures 6 and 7 show several weaker but prominent channels of excitation, which we now consider.

\section{B. Spontaneous two-photon hyper-Raman scattering}

A second and much weaker bound-state oscillation [Fig. 7(b)] is observed at a measured frequency of $\omega_{(b)}=$ $\pm 24 \pm 2 \times 10^{12} \mathrm{rad} / \mathrm{s}(3.8 \pm 0.3 \mathrm{THz})$. In this section, we show that this feature is due to hyper-Raman scattering to the iodine $B$ state, the process shown in Fig. 1(b).

The sinusoidal variation in $Q$ for this mode has a shorter period than the ISRS mode, and therefore, it corresponds to a larger interatomic separation $R_{b}$. The amplitude $A_{b}$ of coherent vibration for this weak mode is not visible in Fig. 5 against the background of the much larger ISRS mode. However, the clear separation between these two processes when the data are displayed as in Figs. 6 and 7 makes it visible over other backgrounds, particularly for $Q>1.5 \AA^{-1}$. This signal can be used to extract the vibrational center of motion, its excursion amplitude, and the phase of its oscillation relative to the time of excitation.

According to Eq. (3) valid for $A_{b}<R_{b}$, the scattering pattern of $\tilde{S}\left(Q, \omega_{b}\right)$ in Figs. 6 and 7 is a higher frequency modulation due to $R_{b}$ contained within a slowly varying envelope determined by $A_{b}$ [18]. The data $\tilde{S}\left(Q, \omega_{b}\right)$ and fit to Eq. (3) are shown in Fig. 10. The best-fit values are $R_{b}=3.10 \pm 0.15 \AA$ and $A_{b}=0.4 \pm 0.4 \AA$. The poor precision for $A_{b}$ is due to the relatively low-momentum cutoff for this scattering energy and the lower signal-to-noise ratio of the signal.

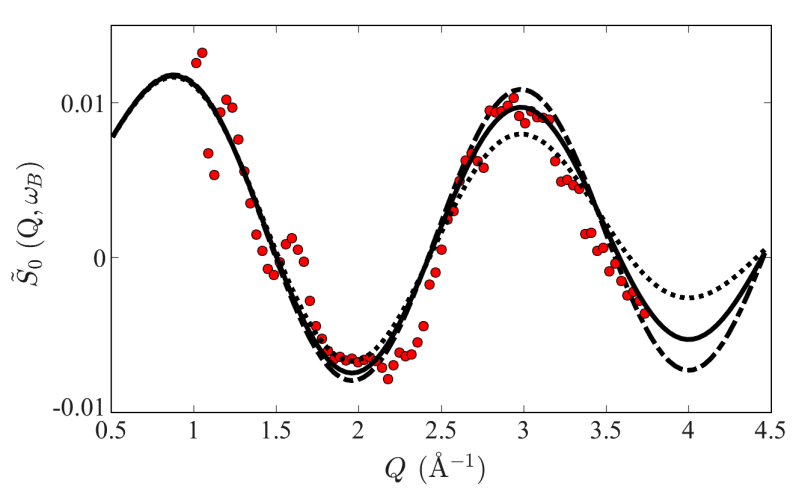

FIG. 10. Dots: $\operatorname{Re}\left[\tilde{S}\left(Q, \omega_{b}\right)\right]$ for $\omega_{b}$ of $24 \pm 2 \times 10^{12} \mathrm{rad} / \mathrm{s}$. Solid line: Fit to Eq. (3) for $A_{b}=0.4 \AA$. Dotted line: Fit for $A_{b}=0.6 \AA$. Dot-dash line: Fit for $A_{b}=0.2 \AA$. The fit parameters are the center of oscillation $R_{b}$, the oscillation excursion $A_{b}$, the scattering background, which produces the offset from zero, and the overall signal amplitude, which can be compared to the signal from ISRS. The value of $R_{b}$ is well constrained by the data: $R_{b}=3.10 \pm 0.15 \AA$. The amplitude of oscillation is much less well constrained, since a factor of 2 change in $A_{b}$ has similar minimum residuals. Based on these values, we assign this oscillation to coherent vibration in the iodine $B$ state at $v=4$. This part of the potential well has a width of $0.4 \AA$ centered on $R=3.1 \AA$. The oscillation frequency for a superposition of $v=4,3$ is $22.6 \times 10^{12} \mathrm{rad} / \mathrm{s}(3.60 \mathrm{THz})[16]$. 
Based on these values, this feature is consistent with coherent excitation of two or more vibrational states centered at $v=4$ in the iodine $B^{1} \Pi_{u}$ state. The average spacing of the iodine atoms, the size of the wave-packet motion, and the oscillation frequency all support this conclusion. The $B$ state lies approximately $2 \mathrm{eV}$ above the iodine ground state, so it is well above resonance for an 800-nm photon and well below resonance for two 800-nm photons. Furthermore, the ungerade symmetry of the $B$ state means that this transition can occur only via interactions with an odd number of photons.

We conclude that the three-photon process of hyperRaman scattering (HRS) (i.e., nonlinear Raman scattering with two photons in and one scattered photon out) is responsible for this excitation [20,21]. This far-off-resonant hyper-Raman scattering occurs during the ultrafast laser pulse, so it can drive vibrational coherences. This process has been studied in other molecular gases and is known to be quite weak, but to our knowledge, it has never been reported in molecular iodine. Nonetheless, our ultrafast x-ray data analyzed to construct $\tilde{S}(Q, \omega)$ are capable of filtering out this weak signal and also provide the amplitude and phase of the resulting molecular motion.

The comparison of the real and imaginary parts of $\tilde{S}\left(Q, \omega_{(b)}\right)$ shows that the phase of the induced oscillation $\delta$ is consistent with zero, indicating that the coherent oscillation of the molecule immediately after excitation is near its inner turning point, not in the middle of its range like the ISRS oscillation. Thus, this weaker oscillation can be seen in $\operatorname{Re}\{\tilde{S}(Q, \omega)\}$, but it is barely visible in $\operatorname{Im}\{\tilde{S}(Q, \omega)\}$ in Fig. 6 .

This independent information about the amplitude of oscillation may now be combined with the ratio of signals in Fig. 7 to estimate the branching ratio between ISRS and HRS for this experiment. The strength of this feature is the product of the fraction of molecules excited to this mode times their squared vibrational excursion [18]. Both processes are quadratic in the incident laser fluence, and so many features of the laser focus cancel out of the ratio. Although the HRS-scattered power is weaker by a factor of 8 , the squared excursion of motion is larger by a factor of nearly 3 , and so the excitation ratio can be estimated to be $\Gamma_{\text {ISRS }} / \Gamma_{\text {HRS }} \approx 20: 1$.

\section{Other vibrations}

The two features that we describe in Secs. III A and III B are the only prominent vibrations in the power spectrum in Fig. 7. One additional vibration, however, is significant by its absence: There is no hint of a molecular cation state formed by ultrafast ionization by six or more $800-\mathrm{nm}$ photons. Ionization should lead to formation of the $\mathrm{I}_{2}^{+}$ cation state $X^{2} \Pi_{3 / 2} g$, which has a significantly contracted potential minimum at $r_{e}=2.58 \AA$ and should produce a vibrational wave packet with angular frequency of
$45 \times 10^{12} \mathrm{rad} / \mathrm{s}$ [22]. This feature is not visible in Fig. 7 . We therefore conclude that multiphoton ionization is extremely small in this experiment.

\section{Two-photon-induced dissociation}

We now turn to two other prominent features in Fig. 7, which are only barely visible in the standard representation of the data in Fig. 5: These are the sloped lines Figs. 7(c) and 7(d), which increase in frequency magnitude as $Q$ increases. A straightforward analysis outlined below shows that these features are due to scattering from dissociation fragments with uniform separation velocities (i.e., ballistic trajectories) [15].

Ballistic dissociation of a pair of molecular fragments that are initially separated by $R_{i}$ has the form

$$
R(\tau)=R_{i}+v \tau
$$

where $v$ is the separation speed, and $R_{i}$ is the initial separation assuming ballistic motion. The projection of the scattering-signal-associated motion onto $P_{l=0}$ is

$$
S_{0}(Q, \tau)=\int d R R^{2} \rho(R, \tau) j_{0}(Q R),
$$

where $j_{0}(Q R)=\sin Q R / Q R$ is the zeroth-order spherical Bessel function, and $\rho$ is the nuclear charge distribution. If we assume for the moment that all the charge is concentrated at a single nuclear separation, i.e., $\rho \propto \delta[R-R(\tau)]$, then the temporal Fourier transform of $S_{0}(Q, \tau)$ contributes a feature to Fig. 7 of this form [15]:

$$
\begin{aligned}
\tilde{S}_{0}(Q, \omega)= & \int_{0}^{\infty} d \tau e^{-i \omega \tau} \frac{\sin Q R(\tau)}{Q R(\tau)} \\
= & \frac{e^{i \omega R_{i} / v}}{2 i Q v} \times\left[E_{1}\left\{-i\left(Q R_{i}-\omega R_{i} / v\right)\right\}\right. \\
& \left.-E_{1}\left\{i\left(Q R_{i}+\omega R_{i} / v\right)\right\}\right] .
\end{aligned}
$$

Here, $E_{1}$ is the exponential integral function [23]. This function has a sharp maximum when the argument approaches zero, and this means that we expect a peak in Fig. 7 along the line $\omega=Q v$, a line with slope $v$ and intercept $Q=\omega=0$. The two sloped lines seen in each half-plane of Fig. 7 are excellent fits to this form, and we find two values of the speed of separation:

$v_{(c)}=14 \pm 1 \AA / \mathrm{ps}$,

$v_{(d)}=23 \pm 1 \AA / \mathrm{ps}$.

The iodine molecule is capable of dissociating following excitation by one $800-\mathrm{nm}$ photon to the $A$ state, but the separation velocity is slow, about $1.7 \AA / \mathrm{ps}$, corresponding to a dissociation energy release of $0.04 \mathrm{eV}$. This velocity falls too close to the $\omega$ axis in Fig. 7 and is also highly dispersed by the shape of the $A$ potential, so it cannot be seen. 
Iodine can absorb two 800-nm photons to repulsive gerade excited states, which dissociate to either ${ }^{2} P_{1 / 2}+{ }^{2} P_{3 / 2}$ or ${ }^{2} P_{3 / 2}+{ }^{2} P_{3 / 2}$ atomic pairs with separation velocities that differ because of the fine-structure splitting in the atomic ground state. The separation speed $v_{3 / 2+1 / 2}=13.4 \AA / \mathrm{ps}$ corresponds to a kinetic energy release of $1.53 \mathrm{eV}$. The speed $v_{3 / 2+3 / 2}=21.6 \AA /$ ps corresponds to a kinetic energy release of $0.59 \mathrm{eV}$.

\section{E. Measured dissociation time shifts}

These nonlinear dissociation channels can be observed using standard particle-detection techniques without the need for $\mathrm{X}$ rays, but ultrafast $\mathrm{X}$ rays provide additional information that is not readily available in other ways. This added information comes from the phase of the dissociation feature in $\tilde{S}_{0}(Q, \omega)$ clearly visible in Fig. 6 and also expressed in Eq. (7). The quantity in square brackets is purely imaginary when the arguments of the two exponential integrals are zero, i.e., at the peak of the dissociationscattering signal. This quantity cancels the $i$ in the denominator, so the overall complex phase of this expression is given by the phase of the exponential prefactor, i.e., $\theta=\omega R_{i} / v$. Since this calculation is done for ballistic motion, we see that $R_{i} / v$ is an effective time $t_{i}$, which corresponds to the back-extrapolated time when the two atoms with constant speed $v$ would have separated from a single point at $R_{i}=0$ if there were no intervening repulsive potential. The value of $t_{i}$ is referenced to the time when the laser pump and the x-ray probe are coincident.

Neither $t_{i}$ nor $R_{i}$ are zero, of course, for the simple reason that the iodine atoms are not colocated when the laser initiates the dissociation, nor do they travel at a uniform speed at first, since they have to escape the molecular potential. The time $t_{i}$ is a kind of "dissociation time shift," a measurable property of the dissociating system, which shows the delay (or advance) suffered by the atoms as they escape the molecular potential. This dissociation time shift is closely related to the "collision lifetime" in potential scattering [24], also sometimes called a "Wigner delay" [25]. A positive value for $t_{i}$ indicates that $\tau=0$ occurs after $t_{i}$, so the molecules are advanced compared to ballistic motion; a negative $t_{i}$ means the dissociation is delayed. Figure 1 shows that the iodine atoms are separated by $2.7 \AA$ when they become excited and gain most of their kinetic energy in the next angstrom of travel, so we expect positive values of $t_{i}$ corresponding to positive dissociation time shifts.

The practical effect of the dissociation time shift can be seen, and also measured, in Fig. 6. The scattering associated with dissociation in $\tilde{S}(Q, \omega)$ is real at $Q=\omega=0$ (out of the range of the measurement), and then the phase advances (or retards) according to the value of $t_{i}$. In the data displayed in the figure, the sign of the real part of the scattering signal indicates that the value of $t_{i}$ is positive, which is what we expect. Furthermore, the second zero appears in the real part of $\tilde{S}$ at $Q=1.8 \AA^{-1}$ for dissociation Fig. 6(c) and at $Q=2.2 \AA^{-1}$ for dissociation Fig. 6(d). This condition implies that $\omega R_{i} / v=Q v t_{i}=+3 \pi / 2$ at that point. Together with our measured exit velocities and combining the errors from these two measurements, we thus recover the dissociation time shifts:

$$
\begin{aligned}
& t_{i(c)}=+188 \pm 20 \mathrm{fs} \\
& t_{i(d)}=+93 \pm 20 \mathrm{fs} .
\end{aligned}
$$

This shift is the time advance observed in the dissociating atoms compared to the time we would measure if they had no interaction and started at the same point. This time is the physical manifestation of two effects: First, the atoms start with a physical separation of $R_{0}>0$. Second, the atoms start nearly at rest, i.e., $v_{i}=0<v$. The net result is the time advance $t_{i}$.

These dissociation time shifts are sensitive to the details of the potentials in the molecule. To show this, we compare the measured time shift with times calculated using classical equations of motion and the known potentials in the molecule [26]. Dissociation channel (d) in Fig. 1 has a measured kinetic energy release that shows it must terminate in the production of a pair of ground-state $\left({ }^{2} P_{3 / 2}\right)$ iodine atoms. This channel is more straightforward to analyze because two-photon absorption from the $X$ state brings the molecule to the vicinity of resonances that correspond to the excitation of the $(2341)^{3} \Pi_{g 1,2}$ dissociative states, as shown in Fig. 1. (Here, "2341" is shorthand denoting the $\sigma_{g}^{2} \pi_{u}^{3} \pi_{g}^{4} \sigma_{u}^{1}$ molecular orbital configuration.) There are no other gerade states in the vicinity.

Employing Mulliken calculations of these potentials, we find that two-photon resonance occurs at an interatomic separation of $R=2.73 \AA$, where there is good FranckCondon overlap with the ground state. A simple integration of the classical equations of motion on this potential with the atoms starting at rest at $R=2.73 \AA$ yields a dissociation time shift of $t_{i}=+102 \mathrm{fs}$, consistent with the measurement.

Dissociation channel (c) in Fig. 1, which must terminate in a ${ }^{2} P_{3 / 2}{ }^{2} P_{1 / 2}$ pair based on the kinetic energy released, is interesting for several reasons. First, there are no gerade dissociative states that terminate in this channel with good Franck-Condon overlap at the point of excitation. The closest is the $(2341)^{1} \Pi_{g}$, which comes into resonance at $R=3.21 \AA$, where the overlap with the ground state is vanishingly small. The calculated dissociation time shift on this potential and from this extended separation is $t_{i}=+183 \mathrm{fs}$, consistent with the measured advance, but there is another possible scenario for this dissociation channel: The molecules detected in dissociation channel (c) in Fig. 1 may have been initially excited to the same Franck-Condon-allowed repulsive potential as those we describe in channel (d). During dissociation, they may couple to the ungerade $B$ state $\left[(2431)^{3} \Pi_{u}\right]$ and climb back 
TABLE I. This table is a summary of all the multiphoton absorption and scattering measurements described in this paper.

\begin{tabular}{|c|c|c|c|}
\hline Process $^{\mathrm{a}}$ & Measurement & Value measured & Calculation or expectation \\
\hline (a) ISRS on the $X$ state & $\begin{array}{l}\text { Center of oscillation } \\
\text { Oscillation frequency } \omega \\
\text { Oscillation amplitude } \\
\text { Oscillation phase } \delta\end{array}$ & $\begin{array}{c}2.79 \pm 0.07 \AA \\
40.3 \pm 1.0 \times 10^{12} \mathrm{rad} / \mathrm{s} \\
0.164 \pm 0.009 \AA \\
290^{\circ}\end{array}$ & $\begin{array}{c}2.67 \AA \text { for } v=1 \rightarrow v=2[28] \\
40.42 \times 10^{12} \mathrm{rad} / \mathrm{s}[16] \\
0.173 \AA \text { for } v=1[17] \\
270^{\circ} \text { (center of oscillation, stretching) }\end{array}$ \\
\hline $\begin{array}{l}\text { (b) } X \rightarrow B \text { Hyper-Raman scattering } \\
\text { (two-photon inelastic scattering) }\end{array}$ & $\begin{array}{l}\text { Center of oscillation } \\
\text { Oscillation frequency } \omega \\
\text { Oscillation amplitude } \\
\text { Oscillation phase } \delta \\
\Gamma_{\mathrm{HRS}} / \Gamma_{\text {ISRS }}\end{array}$ & $\begin{array}{c}3.10 \pm 0.15 \AA \\
24 \pm 2 \times 10^{12} \mathrm{rad} / \mathrm{s} \\
0.4 \pm 0.4 \AA \\
0^{\circ} \\
5 \%\end{array}$ & $\begin{array}{c}3.06 \AA v=4[16] \\
22.6 \times 10^{12} \mathrm{rad} / \mathrm{s} \text { for } v=4,3[16] \\
0.4 \AA \text { for } v=4[16] \\
0^{\circ} \text { (purely displacive) }\end{array}$ \\
\hline $\begin{array}{l}\text { (d) Two-photon dissociation } \\
\text { to the }{ }^{2} P_{3 / 2}-{ }^{2} P_{3 / 2} \text { state }\end{array}$ & $\begin{array}{l}\text { Separation velocity } v_{(d)} \\
\text { Dissociation time shift } \tau_{i(d)}\end{array}$ & $\begin{array}{l}23 \pm 1 \AA / \mathrm{ps} \\
+93 \pm 20 \mathrm{fs}\end{array}$ & $\begin{array}{c}21.6 \AA / p s \\
+102 \text { fs for }{ }^{3} \Pi_{g} \text { dissociation [26] }\end{array}$ \\
\hline $\begin{array}{l}\text { (c) Laser-assisted dissociation } \\
\text { to the }{ }^{2} P_{3 / 2}-{ }^{2} P_{1 / 2} \text { state }\end{array}$ & $\begin{array}{l}\text { Separation velocity } v_{(c)} \\
\text { Dissociation time shift } \tau_{i(c)}\end{array}$ & $\begin{array}{l}14 \pm 1 \AA / \mathrm{ps} \\
+188 \pm 20 \mathrm{fs}\end{array}$ & $\begin{array}{c}13.4 \AA / \mathrm{ps} \\
+204 \mathrm{fs},{ }^{3} \Pi_{g}-{ }^{3} \Pi_{u} \text { dissociation [26] }\end{array}$ \\
\hline
\end{tabular}

a), (b), etc., refer to Figs. 1 and 7.

uphill to the ${ }^{2} P_{3 / 2}{ }^{2} P_{1 / 2}$ dissociation channel. Such $g$ - $u$ couplings are nominally forbidden, but they can occur weakly due to hyperfine mixing [27]. Mixing between gerade and ungerade states is also facilitated by the presence of the external laser field [5]. This pathway has a time advance $t_{i}=+204 \mathrm{fs}$, which is larger than the repulsive potential upper bound because part of this path is along an attractive potential. This channel is also consistent with the measurement.

\section{SUMMARY AND CONCLUSION}

This paper explores ultrafast $\mathrm{x}$-ray detection of twophoton processes in molecules, including impulsive stimulated Raman scattering, hyper-Raman scattering, two-photon photodissociation, and laser-assisted dissociation. The results are summarized in Table I.

Nonlinear photoabsorption in molecules produce many effects that can be accurately measured using the new approach of ultrafast hard x-ray scattering, which has been made possible by the current generation of $\mathrm{x}$-ray free-electron lasers. Some of these phenomena are not detectable at all using all-optical probes. Ultrafast $\mathrm{x}$-ray probes provide de novo information about relative atomic positions, velocities, and accelerations, and so these measurements can reveal information about interatomic forces that can lead to insights to aid both theory and experiments.

These methods may also be extended to photoinduced motion of polyatomic molecules with lighter constituents, especially carbon, nitrogen, and oxygen. The additional nuclear degrees of freedom will lead to a much richer signal. Recent preliminary simulations suggest that FRXS can pick out the modes involved in photoexcitation even when applied to a relatively complex molecule such as alpha-phellandrene (an organic molecule with ten carbons, 16 hydrogens, and dozens of rovibrational modes). In addition, the method can provide information about the relative phase of motion of different modes $[18,29]$. Successful application of these methods to light organic molecules requires high fidelity and high statistics. In this case, the thousandfold increase in the datacollection rate that will be available with next-generation $\mathrm{x}$-ray FELs such as LCLS-II will make such measurements accessible [30].

\section{ACKNOWLEDGMENTS}

We gratefully acknowledge discussions with David Sanchez. This research is supported through the Stanford PULSE Institute, SLAC National Accelerator Laboratory by the U.S. Department of Energy, Office of Basic Energy Sciences, Division of Chemical, Geological and Biosciences, Atomic, Molecular, and Optical Science Program. Use of the Linac Coherent Light Source, SLAC National Accelerator Laboratory is supported by the U.S. Department of Energy, Office of Basic Energy Sciences under Contract No. DE-AC02-76SF00515.

P. H. B. and M. R. W. contributed equally to this work.

[1] S. Ruhman, A. G. Joly, and K. A. Nelson, Time-Resolved Observations of Coherent Molecular Vibrational Motion and the General Occurrence of Impulsive Stimulated Scattering, J. Chem. Phys. 86, 6563 (1987).

[2] S. Ruhman, A. G. Joly, and K. A. Nelson, Coherent Molecular Vibrational Motion Observed in the Time Domain through Impulsive Stimulated Raman Scattering, IEEE J. Quantum Electron. 24, 460 (1988). 
[3] R. W. Terhune, P. D. Maker, and C. M. Savage, Measurements of Nonlinear Light Scattering, Phys. Rev. Lett. 14, 681 (1965).

[4] L. D. Ziegler, Hyper-Raman Spectroscopy, J. Raman Spectrosc. 21, 769 (1990).

[5] P. H. Bucksbaum, A. Zavriyev, H. G. Muller, and D. W. Schumacher, Softening of the $\mathrm{H}_{2}^{+}$Molecular Bond in Intense Laser Fields, Phys. Rev. Lett. 64, 1883 (1990).

[6] T. Zuoand and A. D. Bandrauk, Charge-ResonanceEnhanced Ionization of Diatomic Molecular Ions by Intense Lasers, Phys. Rev. A 52, R2511 (1995).

[7] S. Augst, D. Strickland, D. D. Meyerhofer, S. L. Chin, and J. H. Eberly, Tunneling Ionization of Noble Gases in a HighIntensity Laser Field, Phys. Rev. Lett. 63, 2212 (1989).

[8] B. Stankus, H. Yong, N. Zotev, J. Ruddock, D. Bellshaw, T. Lane, M. Liang, S. Boutet, S. Carbajo, J. Robinson et al., Ultrafast X-Ray Scattering Reveals Vibrational Coherence Following Rydberg Excitation, Nat. Chem. 11, 716 (2019).

[9] M. P. Minitti, J. M. Budarz, A. Kirrander, J. S. Robinson, D. Ratner, T. J. Lane, D. Zhu, J. M. Glownia, M. Kozina, H. T. Lemke et al., Imaging Molecular Motion: Femtosecond $X$-Ray Scattering of an Electrocyclic Chemical Reaction, Phys. Rev. Lett. 114, 255501 (2015).

[10] M. Schmitt, G. Knopp, A. Materny, and W. Kiefer, Femtosecond Time-Resolved Coherent Anti-Stokes Raman Scattering for the Simultaneous Study of Ultrafast Ground and Excited State Dynamics: Iodine Vapour, Chem. Phys. Lett. 270, 9 (1997).

[11] K. Haldrup, G. Levi, E. Biasin, P. Vester, M. G. Laursen, F. Beyer, K. S. Kjær, T. B. van Driel, T. Harlang, A. O. Dohn, M. Sikorski, Y. Feng, S. Nelson, K. Saita, B. Stankus, T. Northey, J. B. Hastings, and P. M. Weber, Ultrafast X-Ray Scattering Measurements of Coherent Structural Dynamics on the Ground-State Potential Energy Surface of a Diplatinum Molecule, Phys. Rev. Lett. 122, 063001 (2019).

[12] J. M. Glownia, A. Natan, J. P. Cryan, R. Hartsock, M. Kozina, M. P. Minitti, S. Nelson, J. Robinson, T. Sato, T. van Driel et al., Self-Referenced Coherent Diffraction X-Ray Movie of Angstrom- and Femtosecond-Scale Atomic Motion, Phys. Rev. Lett. 117, 153003 (2016).

[13] J. M. Glownia, K. Gumerlock, H. T. Lemke, T. Sato, D. Zhu, and M. Chollet, Pump-Probe Experimental Methodology at the Linac Coherent Light Source, J. Synchrotron Radiat. 26, 685 (2019).

[14] M. Ware, J. M. Glownia, A. Natan, J. P. Cryan, and P. H. Bucksbaum, On the Limits of Observing Motion in TimeResolved X-Ray Scattering, Phil. Trans. R. Soc. A 377, 20170477 (2019).

[15] M. R. Ware, J. M. Glownia, N. Al-Sayyad, J. T. O’Neal, and P. H. Bucksbaum, Characterizing Dissociative Motion in
Time-Resolved X-Ray Scattering from Gas-Phase Diatomic Molecules, Phys. Rev. A 100, 033413 (2019).

[16] R. F. Barrowand and K. K. Yee, $B^{3} \Pi_{0+u}-X^{1} \Sigma_{+g}$ System of ${ }^{127} \mathrm{I}_{2}$ : Rotational Analysis and Long-Range Potential in the $B^{3} \Pi_{0+u}$ State, J. Chem. Soc. Faraday Trans. 2 69, 684700 (1973).

[17] R. J. LeRoy, Molecular Constants and Internuclear Potential of Ground-State Molecular Iodine, J. Chem. Phys. 52, 2683 (1970).

[18] M. R. Ware, From Time-Resolved to Frequency-Resolved $X$-Ray Scattering, Ph.D. Thesis, Stanford University, 2019.

[19] D. W. Broege, R. N. Coffee, and P. H. Bucksbaum, StrongField Impulsive Alignment in the Presence of High Temperatures and Large Centrifugal Distortion, Phys. Rev. A 78, 035401 (2008).

[20] S. J. Cyvin, J. E. Rauch, and J. C. Decius, Theory of HyperRaman Effects (Nonlinear Inelastic Light Scattering): Selection Rules and Depolarization Ratios for the SecondOrder Polarizability, J. Chem. Phys. 43, 4083 (1965).

[21] A. M. Kelley, Hyper-Raman Scattering by Molecular Vibrations, Annu. Rev. Phys. Chem. 61, 41 (2010).

[22] L.-h. Deng, Y.-y. Zhu, C.-1. Li, and Y.-q. Chen, HighResolution Observation and Analysis of the $\mathrm{I}_{2}^{+} A^{2} \Pi_{3 / 2, u-} X^{2} \Pi_{3 / 2, g}$ System, J. Chem. Phys. 137, 054308 (2012).

[23] M. Abramowitz, Handbook of Mathematical Functions, with Formulas, Graphs, and Mathematical Tables (Dover Publications, Inc., New York, 1974).

[24] F. T. Smith, Lifetime Matrix in Collision Theory, Phys. Rev. 118, 349 (1960).

[25] E. P. Wigner, Lower Limit for the Energy Derivative of the Scattering Phase Shift, Phys. Rev. 98, 145 (1955).

[26] R. S. Mulliken, Iodine Revisited, J. Chem. Phys. 55, 288 (1971).

[27] J. P. Pique, F. Hartmann, R. Bacis, S. Churassy, and J. B. Koffend, Hyperfine-Induced Ungerade-Gerade Symmetry Breaking in a Homonuclear Diatomic Molecule near a Dissociation Limit: ${ }^{127} \mathrm{I}_{2}$ at the ${ }^{2} P_{3 / 2}-{ }^{2} P_{1 / 2}$ Limit, Phys. Rev. Lett. 52, 267 (1984).

[28] W. F. Howard and L. Andrews, Matrix Raman Spectra of the Molecular Halogens: Resonance Raman Spectra of Isolated and Aggregated $\mathrm{I}_{2}$, J. Raman Spectrosc. 2, 447 (1974).

[29] M. Sanchez and D. M. Ware, Preliminary Calculations (to be published).

[30] R. Schoenlein, P. Abbamonte, F. Abild-Pedersen, P. Adams, M. Ahmed, F. Albert, R. A. Mori, A. Anfinrud, A. Aquila, M. Armstrong et al., New Science Opportunities Enabled by LCLS-II X-Ray Lasers, SLAC Report (2015). 